\title{
A 1.5V CMOS High-Speed 16-bit $\div 8$-bit Divider Using the Quotient-Select Architecture and True-Single-Phase Bootstrapped Dynamic Circuit Techniques Suitable for Low-Voltage VLSI
}

\author{
C. C. Yeh, J. H. Lou and J. B. Kuo \\ Rm. 338, Dept. of Electrical Eng., National Taiwan University \\ Roosevelt Rd., Sec. 4, \#1, Taipei, Taiwan 106-17
}

\begin{abstract}
This paper reports a $1.5 \mathrm{~V}$ high-speed 16-bit $\div 8$-bit divider circuit using the quotient-select architecture and true-single-phase bootstrapped dynamic circuit techniques. Based on a $0.8 \mu \mathrm{m}$ CMOS technology, the speed performance of this 16 -bit $\div 8$ bit divider circuit is improved by $\mathbf{4 5 \%}$ as compared to the divider using the non-restoring iterative architecture and the domino dynamic logic circuits without the bootstrapped technique.
\end{abstract}

\section{INTRODUCTION}

Division is an important function in a CPU arithmetic unit. Enhancing the speed performance of a divider circuit is critical in raising the speed performance of a VLSI CPU [1]-[3]. Fig. 1 shows the block diagram of a 16bit $\div 8$-bit divider circuit using a non-restoring iterative architecture [1]. A 16-bit dividend and an 8-bit divisor are assumed to be positive and smaller than 1 . As in a standard binary division operation, successively right-shifted values of the divisor are subtracted from or added to the the dividend. Also, for next-generation deep-submicron CMOS VLSI technology, low supply voltage is the trend. For sub-0.1 $\mu \mathrm{m}$ CMOS technology, $1.5 \mathrm{~V}$ is necessary. At a supply voltage of $1.5 \mathrm{~V}$, the speed performance of CMOS dynamic logic circuits such as NORA [4], domino, Zipper is better than that of CMOS static ones as a result of reduced internal parasitic capacitances. However, as the serial fan-in is large, its associated propagation delay may increase drastically, which is especially serious at a low supply voltage. In this paper, by using a $1.5 \mathrm{~V}$ CMOS dynamic logic circuit with a bootstrapper technique, a $1.5 \mathrm{~V}$ high-speed CMOS bootstrapped 16-bit $\div 8$-bit divider using the quotient-select architecture is reported. It will be shown that the speed performance of this 16 -bit $\div 8$ bit divider circuit is improved by $45 \%$ as compared to the divider using the non-restoring iterative architecture and the domino dynamic logic circuits without the bootstrapped technique.

This work is supported under R.O.C. National Science Council Contracts \#84-2622-E002-008, 011 \& \#85-2215-E0002-024.
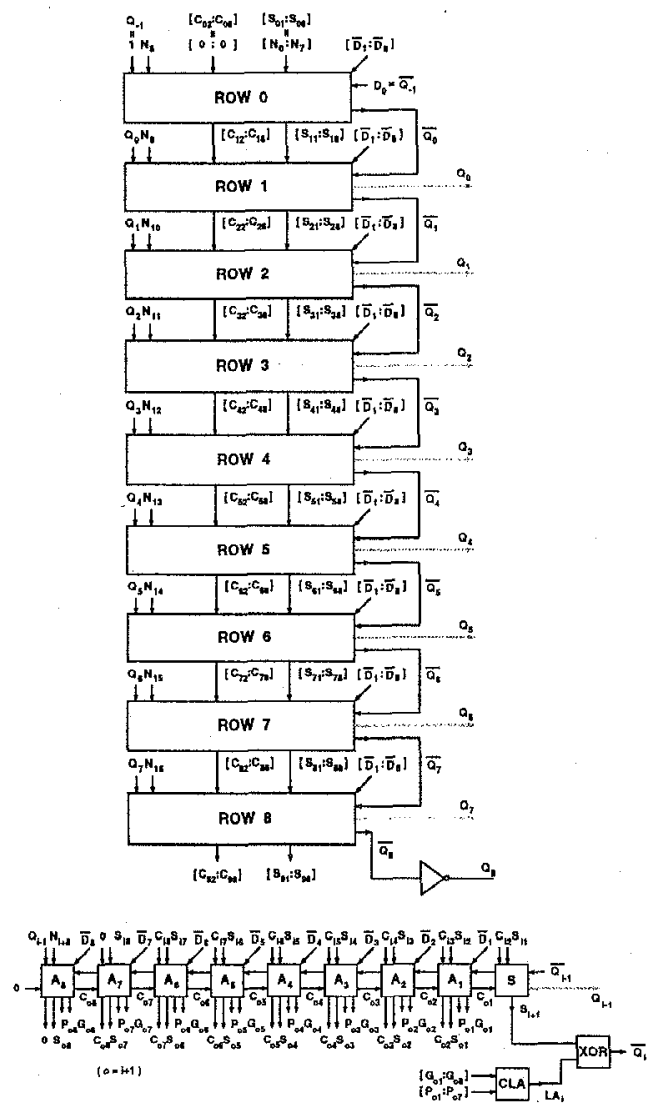

Fig. 1. The architecture of a 16 -bit $\div 8$-bit non-restoring iterative divider.

\section{Conventionat Non-Resoring Iterative DIVIDER}

In a conventional 16 -bit $\div 8$-bit non-restoring iterative divider, eight 'quo tient rows' are required. In each quotient row, there are $\mathrm{A}$ (adder) cells, an S (sign) cell, and a CLA (carry look-ahead) cell. Each A cell is composed of a full adder and a control signal implemented by an EXCLUSIVE-OR gate. The control signal decides whether an add or a subtract to be performed. In the jth A cell in the (i-1)th $r$ ow, using a full adder, its associated sum and carry signals at the output are related to the sum and carry signals of the previous row as:

$$
\begin{aligned}
S_{i, j}= & \left(\bar{Q}_{i-2} \oplus \bar{D}_{j}\right) \oplus S_{i-1, j+1} \oplus C_{i-1, j+2} \\
C_{i, j}= & \left(\bar{Q}_{i-2} \oplus \bar{D}_{j}\right)\left(S_{i-1, j+1}+C_{i-1, j+2}\right) \\
& +S_{i-1, j+1} C_{i-1, j+2},
\end{aligned}
$$



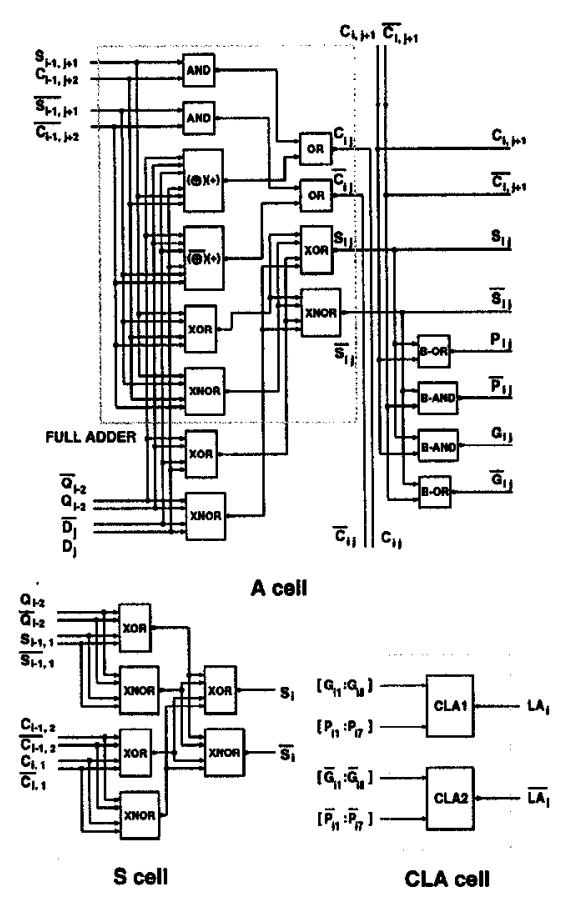

Fig. 2. The functional blocks of the ith quotient row in the 16bit $\div 8$-bit non-restoring iterative divider.

where $\bar{Q}_{i-2}$ is the negative of the (i-2)th-bit quotient, and $\bar{D}_{j}$ is the negative of the jth-bit dividend. In addition, propagate and generate signals for the carry look-ahead circuit have been produced:

$$
\begin{aligned}
P_{i, j}= & {\left[\left(\bar{Q}_{i-2} \oplus \bar{D}_{j}\right) \oplus S_{i-1, j+1} \oplus C_{i-1, j+2}\right] } \\
& +C_{i, j+1}, \\
G_{i, j}= & {\left[\left(\bar{Q}_{i-2} \oplus \bar{D}_{j}\right) \oplus S_{i-1, j+1} \oplus C_{i-1, j+2}\right] } \\
& \cdot C_{i, j+1} .
\end{aligned}
$$

The S cell, which contains the sum portion of the A cell, is used to compute the sign for each row:

$$
S_{i}=\left(\bar{Q}_{i-2} \oplus S_{i-1,1} \oplus C_{i-1,2}\right) \oplus C_{i, 1} .
$$

Eqs. (1)-(4) are applicable for the cells not at the top and left boundaries $\left(2 \leq i \leq b_{q}+1\right.$ and $\left.1 \leq j \leq b_{d}-1\right)$. For the cells at the top and left boundaries $(i=1$ or $j=b_{d}$ ), where $b_{q}$ is the quotient bit number, and $b_{d}$ is the divisor bit number, Eqs.(1)-(4) should be modified to include appropriate boundary conditions as shown in Fig. 1. Note that $N_{i}$ is the ith bit dividend. As shown in Fig. 2, the CLA cell is used to compute the final carry signal according to the following formula:

$$
\begin{aligned}
Y_{i, j} & =G_{i, j}+P_{i, j} Y_{i, j+1}, \quad j=1, \ldots, b_{d} \\
L A_{i} & =Y_{i, 1} .
\end{aligned}
$$

Using an EXCLUSIVE-OR logic gate, the ith quotient bit at the output is low if either output from the $\mathrm{S}$ cell or $\mathrm{t}$ he CLA cell is 1 .

The speed performance of a non-restoring iterative divider is determined by the speed of the propagate and

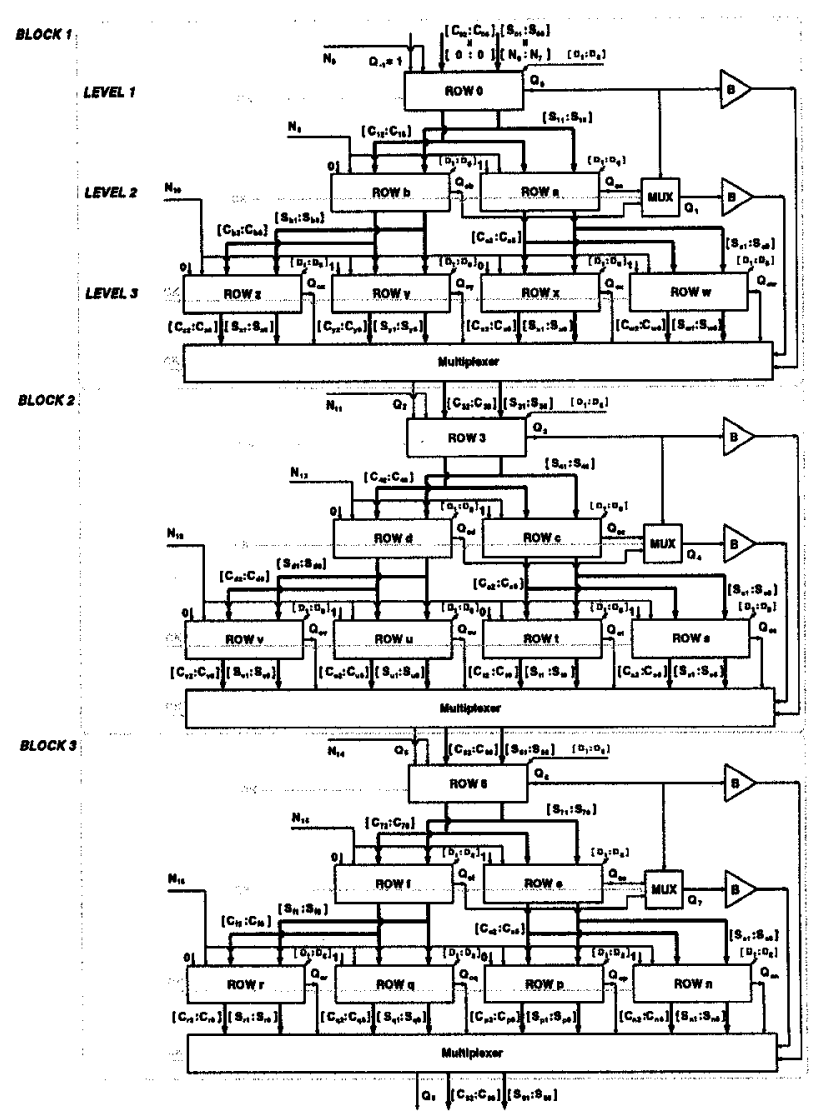

Fig. 3. Block diagram of the $1.5 \mathrm{~V} 16$-bit $\div 8$-bit divider using the quotient-select architecture and true-single-phase bootstrapped dynamic circuit techniques.

generate signals of the A-cells, the delay time of the $L A_{i}$ signal in the CLA cell and the speed of producing the quotient bit $\overline{Q_{i}}$ of the XOR in the quotient row. After the quotient bit of a quotient row $\left(\overline{Q_{i}}\right)$ is produced, its value is transferred to the next row. Then, the quotient bit of the next row $\left(\overline{Q_{i+1}}\right)$ is computed. No quotient bit of the next row can be computed until the quotient bit of the previous row is obtained.

\section{The Parallel-Out Quotient-Select Divider}

As shown in Fig. 3, in the 3-bit parallel-out quotientselect architecture, instead of waiting for the quotient bit from the previous row, three quotient blocks have been used to produce the nine output quotient bits almost simultaneously. In each block, three levels of quotient rows have been arranged. For example, under the quotient row of the first level, at the second level there are two quotient rows: $a$ and $b$. At the third level, there are four quotient rows: $w, x, y$, and $z$. The second and the third levels of quotient rows have been arranged to produce three output quotient bits: $Q_{0}, Q_{1}$, and $Q_{2}$ simultaneously. In these seven quotient rows in three levels, the input quotient bits of rows $0, a, b, w, x, y$, and $\mathrm{z}$ have been designated as 1 , $1,0,1,0,1$, and 0 , respectively. 


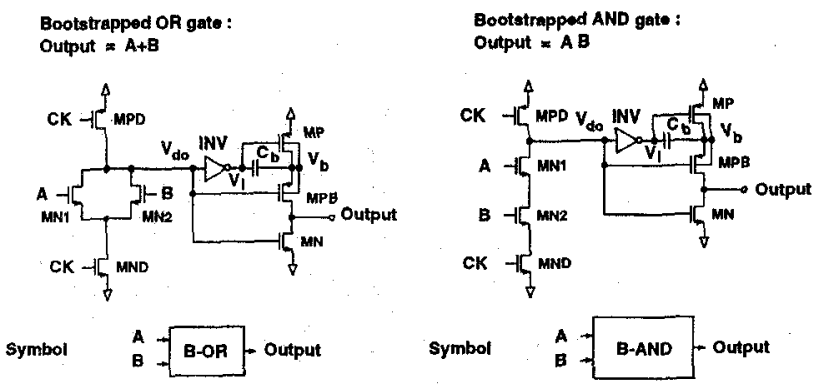

Fig. 4. The 1.5V CMOS bootstrapped dynamic logic circuits including the CMOS bootstrapper circuit.

The seven individual output quotient bits of each row in three levels are $Q_{0}, Q_{o a}, Q_{o b}, Q_{o w}, Q_{o x}, Q_{o y}$, and $Q_{o z}$, respectively. The inputs to the first block are the dividend bits: $N_{0}-N_{8}$. The output quotient bit of row $0-Q_{0}$ may be 0 or 1 . The sum and the carry signals produced by row 0 are transferred to rows $\mathrm{a}$ and $\mathrm{b}$ such that $Q_{o a}$ and $Q_{o b}$ can be computed immediately without waiting for the generation of $Q_{0}$. Another dividend bit $N_{9}$ is used as the input to both the quotient rows a and $\mathrm{b}$. Then, the output quotient bit- $Q_{1}$ is equal to $Q_{o a}$ or $Q_{o b}$ depending on $Q_{0}$ by the multiplexer-MUX. If $Q_{0}$ is 1, MUX outputs $Q_{o a}$ as $Q_{1}$. If $Q_{1}$ is 0, MUX outputs $Q_{o b}$ as $Q_{1}$. The outputs of the second-level quotient rows- $S_{a 1}-S_{a 8}$, $C_{a 2}-C_{a 8} ; S_{b 1}-S_{b 8}, C_{b 2}-C_{b 8}$ are used as inputs to the third level. In addition, another dividend bit $N_{10}$ is used as an input to the third level.

The output quotient bit $Q_{2}$ of the third level is determined by a similar decision criterion as in the second level. Under the third level of the quotient rows, a multiplexer is used to select the sum and the carry of the first block$S_{32}-S_{38}, C_{31}-C_{38}$. The second block uses the outputs from the first block to generate the output quotient bits: $Q_{3}$, $Q_{4}$, and $Q_{5}$. The third block generates the output quotient bits: $Q_{6}, Q_{7}$, and $Q_{8}$.

The speed of the 16 -bit $\div 8$-bit divider with the quotientselect architecture is determined by the propagation delay of each of the three blocks as shown in Fig.3. The propagation delay of producing the output quotient bits $Q_{0}$, $Q_{1}$, and $Q_{2}$ of the first block is mainly determined by the propagati on delay of the sum and the carry signals $\left(S^{\prime} s\right.$, $C^{\prime} s$ ) associated with each quoti ent row in all three levels in the first block. Although there are three levels in the first block, the speed of producing $Q_{1}$ and $Q_{2}$ is not substantially slower than that of producing $Q_{0}$ since the critical component of the propagation delay in producing the three output quotient bits- $Q_{0}, Q_{1}$ and $Q_{2}$ is on the adder circuit in the A cell. Therefore, the speed of generating $Q_{0}, Q_{1}$ and $Q_{2}$ is about identical. Similar situations exist for $Q_{3}, Q_{4}$ and $Q_{5}$ for the second block and $Q_{6}, Q_{7}$ and $Q_{8}$ for the third block.

As a result, the speed of the 16 -bit $\div 8$-bit divider with the quotient-select architecture is about three times faster

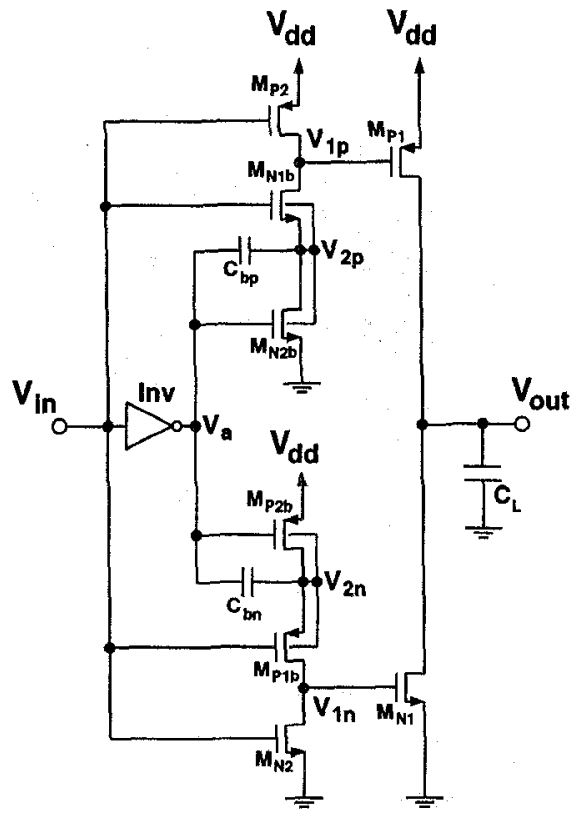

Fig. 5. The 1.5V CMOS buffer circuit using the bootstrapper technique.

as compared to that with the conventional non-restoring iterative architecture. Fig. 4 shows the $1.5 \mathrm{~V}$ CMOS bootstrapped dynamic logic circuits including the CMOS bootstrapper circuit. As CK is low, it is the precharge period of bootstrapper circuits. During the precharge period, the internal node $\left(V_{d o}\right)$ is precharged to $V_{d d}$, and the output voltage $V_{\text {out }}$ is predischarged to ground via $M N$. The bootstrap capacitor $\left(C_{b}\right)$ is charged to $V_{d d}$ - the left side is grounded and the right side is at $V_{b}=V_{d d}$. During the precharge period, the right side of the bootstrap capacitor is separated from the output since MPB is off. As CK turns high, it's the logic evaluation period. During the logic evaluation period, MPD, MP, M N turn off. During the logic evaluation period, the internal node voltage $V_{d o}$ is determined by inputs $A$ and $B$. If both $A$ and $B$ are high, $V_{d o}$ is pulled low and $V_{I}$ is high. Owing to the charge in the bootstrap capacitor, $V_{b}$ will be bootstrapped to over $V_{d d}$ - the internal voltage overshoot. In addition, as MPB turns on, $V_{\text {out }}$ is pulled high to over $V_{d d}$. In the CLA cell, owing to the $1.5 \mathrm{~V}$ bootstrapped CMOS dynamic logic circuit, the signal swing of the input signals $-P^{\prime} s$ and $G^{\prime} s$ exceeds $2 \mathrm{~V}$. As a result, the switching speed of the CLA cell is enhanced.

Fig. 5 shows the $1.5 \mathrm{~V}$ CMOS buffer (B) circuit using the bootstrapper technique[ 5]. During the pull-up transient, the operation of the full-swing bootstrapped CMOS buffer circuit is divided into two periods regarding the bootstrap capacitor $\mathrm{C}_{\mathrm{bp}}$ : (1) the charge build-up period and (2) the bootstrap period. Prior to the pull-up transient, the input is at $0 \mathrm{~V}$ and at the output of the inverter $\mathrm{V}_{\mathrm{a}}$ is at $1.5 \mathrm{~V}$. Therefore, $\mathrm{M}_{\mathrm{N} 1 \mathrm{~b}}$ and $\mathrm{M}_{\mathrm{N} 2}$ are off $; \mathrm{M}_{\mathrm{N} 2 \mathrm{~b}}$ is on. At the output of the buffer, $V_{\text {out }}$ is at $0 \mathrm{~V}$. On the other hand, $\mathrm{M}_{\mathrm{N} 2 \mathrm{~b}}$ and $\mathrm{C}_{\mathrm{bp}}$ of the bootstrap segment are separated from the $M_{P 2}$ and $M_{P 1}$ of the fundamental seg- 


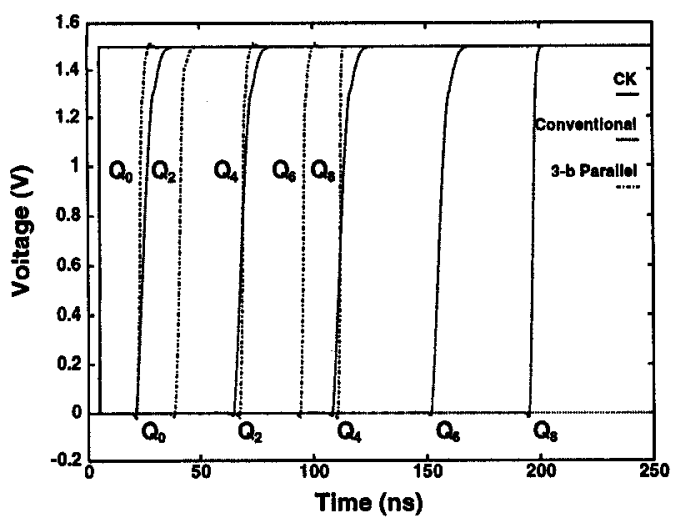

Fig. 6. Transient waveform of the $1.5 \mathrm{~V} 16$-bit $\div 8$-bit divider using the quotient-select architecture and the true-single-phase bootstrapped dynamic circuit techniques.

ment. As a result, the bootstrap capacitor $\mathrm{C}_{\mathrm{bp}}$ has charge of $1.5 \mathrm{C}_{\mathrm{bp}}$ Coulomb After the input ramp-up period, the right side of the bootstrap capacitor $\mathrm{C}_{\mathrm{bp}}$ is disconnected from ground since $\mathrm{M}_{\mathrm{N} 2 \mathrm{~b}}$ is off. Instead, it's connected to the gate of $M_{P_{1}}$ since $M_{N 1 b}$ is on. Due to the voltage change at the left side of the bootstrap capacitor $\mathrm{C}_{\mathrm{bp}}$, the right side of the bootstrap capacitor $\mathrm{C}_{\mathrm{bp}}$ changes to below $0 \mathrm{~V}$ - the internal voltage undershoot. As a result, the output voltage can switch at a faster pace since the gate of $\mathrm{M}_{\mathrm{P} 1}$ is driven at below $0 \mathrm{~V}$. Pull-down transient has a complementary configuration.

\section{Results and Discussion}

Fig. 6 shows the transient waveform of the $1.5 \mathrm{~V} 16-$ bit $\div 8$-bit divider using the quotient-select architecture and the true-single-phase bootstrapped dynamic circuit techniques. The load at the quotient bit output is $0.1 \mathrm{pf}$. At a supply voltage of $1.5 \mathrm{~V}$, the propagation delay of the output quotient bit $Q_{8}$ is $107 \mathrm{~ns}$ for the 16-bit $\div 8$ bit divider using the 3-bit parallel-out quotient-select architecture. Compared with the propagation delay of the divider using the conventional non-restoring iterative architecture $-192 \mathrm{~ns}$, a speed enhancement of $1.8 \mathrm{x}$ has be en reached, which is less than $3 \mathrm{x}$ as expected. This is due to the fact that the quotient-select architecture is not fully "parallel-processing". The propagation delays of two consecutive quotient rows of a block are differed by the delay in a full adder. In addition, the extra delay due to the multiplexer and the buffer also contributes to the shrinkage in the speed enhancement.

Fig. 7 shows the delay time vs. supply voltage of the true-single-phase CMOS boo tstrapped divider. As shown in the figure, regardless of the supply voltage, a consistent improvement in the speed for the 16-bit $\div 8$-bit divider using the 3-bit parallel-out quotient-select architecture over the the one using the conv entional non-restoring iterative architecture can be seen.

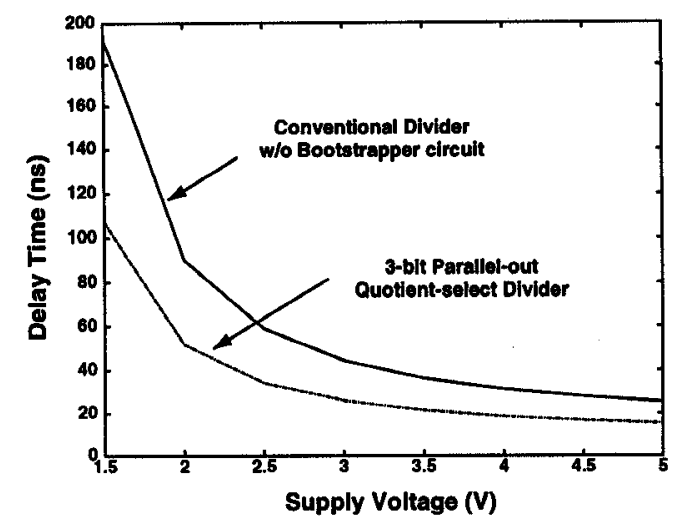

Fig. 7. Delay time vs supply voltage of the true-single-phase CMOS bootstrapped divider.

\section{Conclusion}

In this paper, a 3-bit parallel-out quotient-select architecture has been studied. In fact, for a large-size divider system such as a 64 -bit $\div 32$-bit divider, a 8-bit parallelout quotient-select architecture can be used to further enhance the speed performanc e. The more bits used in the parallel-out quotient-select structure, the more improvement in speed can be expected. However, a larger die area is also needed.

\section{REFERENCES}

[1] M. Cappa and V. C. Hamacher, "An Augmented Iterative Array for High-Speed Binary Division," IEEE Trans. Computers, Vol. C-22, No. 2, pp.172-175, Feb. 1973.

[2] R. Stefanelli, "A Suggestion for a High-Speed Parallel Binary Divider," IEEE Trans. Computers, Vol. C-21, No. 1, pp.4255 , Jan 1972.

[3] A. Vandemeulebroecke, E. vanzieleghem, T. Denayer, and P. G. A. Jespers, "A New Carry-Free Division Algorithm and its Applications to a Single-Chip 1024-b RSA Processor, " IEEE J. Solid-State Circuits, Vol. 25, No. 3, pp. 748-756, June 1990.

[4] N. F. Gonzales and H. 'J. DeMan, "NORA: A Racefree Dynamic CMOS Technique for Pipelined Logic Structures," IEEE J. Solid-State Circuits, Vol. 18, pp. 261-266, June 1983.

[5] J. H. Lou and J. B. Kuo, "A 1.5V Full-Swing Bootstrapped CMOS Large Capacitive-Load Driver Circuit Suitable for LowVoltage CMOS VLSI," IEEE J. Solid-State Circuits, Vol. 32, No. 1, pp. 119-121, Jan. 1997. 